\section{Arquitectura como materialización de un concepto. La espacialidad Megalítica}

Felipe Criado Boado, Patricia Mañana BorrazÁs Laboratorio de Arqueoloxía, Instituto de Estudios Gallegos Padre Sarmiento (CSIC-XuGa) / UA LaFC (IIT, USC)

\section{Resumen}

Abordamos la arquitectura como uno de los aspectos de una cultura en la que de manera más directa una sociedad imprime su manera de estar en el mundo, cómo quiere que sea y cómo lo modifica para crear un entorno acorde con su pensamiento. Por lo tanto, partiendo de que la arquitectura es un producto social, el análisis de una construcción según su propia lógica nos debe permitir acceder de alguna manera a ese pensamiento, del que no tenemos otras evidencias en sociedades de las que no tenemos individuos vivos o textos escritos que la forma en que lo construyeron, cómo lo configuran formalmente. Así, explicaremos las bases teóricas y metodológicas de esta perspectiva y cómo es posible acceder al código espacial de una sociedad a través de ella.

Palabras Clave: Arqueología de la Arquitectura; Arqueología del Paisaje; patrón de racionalidad; neolítico; espacio construido; túmulo.

\footnotetext{
Abstract

We approach architecture as one of the aspects of a culture in which a society directly imposes its way of being on the world, how it wants the world to be, and how it modifies it to create an environment in line with its philosophy. Therefore, working from the basis that architecture is a social product, the analysis of a construction according to its own logic should allow us to somehow access this philosophy, of which we have no other evidence in societies where there are no living individuals or written texts other than the way they built and formally shaped this philosophy. In this way we can explain the theoretical and methodological bases of this perspective and how it is possible to access the spatial code of a society through this.
}

Key words: Archaeology of Architecture; Landscape Archaeology; rationality pattern; Neolithic; constructed space; burial mound.

\section{UN PROGRAMA DE INVESTIGACIÓN ARQUEOLÓGICA SOBRE EL PENSAMIENTO}

Presentamos un modo de analizar el registro arquitectónico cuyo fin último es acceder a la racionalidad de las sociedades que han construido y el significado los espacios construidos. La perspectiva que se ha asumido como punto de partida se inscribe dentro de la estrategia de investigación desarrollada por el Laboratorio de Arqueoloxía del Instituto de Estudios Galegos Padre Sarmiento (CSIC-XuGa) vertebrada en torno a la Arqueología del Paisaje, un programa de investigación de raigambre estructuralista que incide en la espacialidad de las sociedades pretéritas que analiza, entendiendo que el espacio (la forma de configurarlo, de construirlo) es uno de los aspectos en los que una comunidad mejor se reproduce, se representa a sí misma (CRIADO, 1999, 2002, MAÑANA et alii., 2002) y por lo tanto, su análisis nos ha de permitir acceder de alguna manera a cómo era esta sociedad.

Por lo tanto, el objetivo primordial es analizar, reconstruir e interpretar los paisajes arqueológicos a partir de los elementos que los concretan, es decir, analizar de manera integral los procesos y formas de culturización del espacio a lo largo de la historia, comprendidos como entidades espaciales y fenómenos sociales y no como hechos aislados. Se parte de que las actividades que tienen lugar en relación con el espacio, están organizadas de forma coherente con la representación ideal del mundo que tiene el grupo social que las realiza, es decir, que en el proceso de construcción de los espacios intervienen no sólo los dispositivos mecánicos (físicos) sino que incluyen también los dispositivos conceptuales (que definen, articulan y nombran), necesarios para poder llevar a cabo la "humanización» de un espacio. Analizando estos dispositivos mecánicos (las formas), su configuración, los cambios según el distinto tipo de sociedad, etc., se debería poder llegar a acceder en cierta medida a los dispositivos conceptuales que los han generado, en definitiva, a su patrón de racionalidad (CRIADO, 1999: 10).

Entendemos además que los hechos formales que definen una sociedad se pueden modelizar tras un proceso de deconstrucción, en el que se trata de describir al elemento formal por sus propios parámetros, sin introducir un sentido extraño (el del arqueólogo) a él. Es una práctica arqueológica que entiende que todas las representaciones de una sociedad, incluida las acciones a nivel individual, dependen de una estructura subyacente, de una misma racionalidad, que permite a todas esas distintas acciones existir. Así, cualquier acción con evidencias materiales (que son de las que queda registro arqueológico), incluso si ésta no es hecha de manera intencional, es ante todo un valor cultural, o sea, es ante todo una acción que no sería viable si no estuviera 
dentro de los límites que marca esa racionalidad: lo que se identifica en el registro arqueológico es ante todo una materialización del pensamiento, construcciones en las que una sociedad produce y reproduce su realidad (CRIADO 1999, 2002).

Esta es una concepción que dimana de Foucault, en el que se identifican varias derivaciones útiles para la Arqueología (CRIADO, 1993): espacio, pensamiento y sociedad están íntimamente ligados, siendo la construcción del espacio una parte fundamental de la construcción de la realidad de un determinado sistema de saber-poder. Es importante reconocer que el espacio no es un escenario estático, ya dado, sino que se constituye como una construcción social, imaginaria, en movimiento y enraizada en la cultura, hallándose en estrecha relación pensamiento, organización social, subsistencia, y concepción y uso del espacio.

La investigación parte del presupuesto teórico de la multidimensionalidad del paisajelespacio, por la cual el paisaje / espacio construido se encuentra constituido por tres dimensiones o niveles distintos (CRIADO, 1996a: 17, 1999: 6):

-El espacio en cuanto entorno fisico o matriz medioambiental sobre la que los hombres realizan sus actividades.

-El espacio en cuanto entorno social o medio construido por el ser humano, en el que se producen las relaciones entre individuos y grupos.

-El espacio en cuanto entorno pensado o medio simbólico que ofrece la base para comprender la apropiación humana de la naturaleza.

De acuerdo con este marco teórico y conceptual, la Arqueología del Paisaje prioriza el estudio del espacio, analizado y pensado a través del registro empírico, pero a diferencia de la Arqueología Espacial, integrando en este estudio la parte imaginaria-simbólica del mismo, es decir, intenta elaborar modelos de interrelaciones entre los tres tipos de espacios definidos y articular el análisis complementario de estas tres dimensiones del espacio, tratando de no centrarse en una de ellas como representación de la globalidad de la concepción espacial.

Esta definición previa al desarrollo de las técnicas analíticas, implica la necesidad de que el análisis arqueológico se deba aplicar en los distintos ámbitos en los que un fenómeno cultural se significa, en sus diferentes dimensiones: económica (subsistencia, explotación de los recursos), social (emplazamiento, monumentalidad, territorialidad) y simbólica, dimensiones que se reflejan en cada uno de los productos materiales de una formación social (hábitat, arquitectura, arte, cerámica, ...). Cada uno de estos ámbitos está determinado por códigos espaciales compatibles y semejantes entre sí, presentando relaciones de compatibilidad y configurando una regularidad espacial, ya que obedecen a la misma estrategia de construcción del espacio social, al mismo patrón de organización, a la misma racionalidad.

De este modo, el espacio arquitectónico se puede definir como un producto humano que utiliza una realidad dada (el espacio físico) para crear una realidad nueva: el espacio construido y, por consiguiente, social ${ }^{1}$, al que se confiere un significado simbólico. Dicho producto se compone de diferentes entidades formales, que se proyectan espacialmente, son visibles, por lo que pueden ser percibidas y descritas por la observación arqueológica.

Para llegar a profundizar en la sociedad que los construyó, además que estudiar los tipos de materiales, la forma de colocación o las técnicas constructivas, es fundamental definir cómo se han organizado estos volúmenes y qué espacios han sido formados, o bien qué recursos (simbólicos) se han empleado para crear un determinado escenario, ambiente en el que se desenvuelven las relaciones sociales a todos los niveles, desde las relaciones individuales, familiares hasta las más generales (intergrupales) ${ }^{2}$. El análisis de las relaciones espaciales significativas entre las entidades del registro, permite reconstruir mínimamente su contexto y, en menor medida, su sentido original. El estudio de estas relaciones espaciales entre elementos, esto es, la estructura espacial, permite acercarnos no sólo a la lógica espacial de una determinada comunidad, en este caso el conjunto de relaciones interespaciales que construyó una sociedad, sino también a la propia lógica social de ese espacio (HILlier, HANSON, 1984). Es un producto cultural destinado a comunicar una información que es manejada, consciente e inconscientemente, por el colectivo que la construye; se trata de un espacio físico en el que se desarrolla, reproduciéndola a la vez, la acción social prehistórica (SHANKS, TilleY, 1987). La forma de la construcción, del espacio construido, aporta un medio perdurable para imponer esquemas de organización social, es tanto un reflejo como un generador activo de conducta social, de ahí que no sólo deba ser interpretada únicamente en términos funcionales, sino también en términos sociales (LOCOCK, 1994).

En una sociedad de la que no existe registro escrito, de la que no permanecen sujetos que hablen y comuniquen su pensamiento, es muy difícil acceder a esa parte simbólica que por lo que se ha propuesto, consideramos parte fundamental de la concepción espacial y del patrón de racionalidad. Uno

\footnotetext{
${ }^{1}$ Se trataría de superar el paradigma actual que conceptualiza el espacio como un ente sin contenido social y la sociedad como un ser sin contenido espacial.

2 Creemos que esta temática se debe incluir como un aspecto más de la cadena tecnológica, y no reducir esta a los conjuntos de técnicas, procedimientos y herramientas que se utilizan en un proceso de producción; tal y como plantean Cobas y Prieto 2002.
} 
de los presupuestos básicos de la Arqueología del Paisaje que ahonda en este punto es que todo lo visible es simbólico (Criado, 1993, 1999); se plantea que forma parte de una racionalidad «una voluntad de hacer que los procesos sociales y/o sus resultados sean más o menos visibles o invisibles a nivel social y que esto es así porque las condiciones de visibilidad de los resultados de la acción social son de hecho la objetificación de la concepción espacial vigente dentro del contexto cultural en el que se desarrolla esa acción» (Criado, 1993:42-3). Esta voluntad de visibilidad puede ser tanto consciente y explícita como implícita e incluso inconsciente: es la racionalidad de un grupo social la que determina qué rasgos de ese grupo serán visibles.

Sobre esta base teórica y metódica, se articulan las herramientas metodológicas que nos permiten construir conocimiento nuevo, tratando con ello de acceder al sentido original de los espacios construidos. El objetivo en este análisis, de base estructuralista, es buscar las regularidades formales que definen un fenómeno o sociedad.

La herramienta metodológica primordial de esta propuesta es el análisis formal. Aporta un procedimiento analítico que permite $\left(1 .{ }^{\circ}\right)$ desconstruir y $\left(2 .^{\circ}\right)$ describir los fenómenos considerados, sin introducir un sentido extraño a ellos. Ésta es una técnica fundamental en esta metodología y un objetivo en sí mismo, porque cuando tiene éxito, describe el objeto de estudio desde sí mismo, eliminándose factores más subjetivos que cambian el significado de lo estudiado. La deconstrucción implica la descomposición del espacio social en los niveles que lo constituyen, con el fin de identificar cuáles son los elementos básicos que lo conforman y descubrir su morfología y configuración interna, tratando de evitar que el estudio reproduzca los rasgos del horizonte de racionalidad del estudioso. Con este tipo de análisis se pretende llegar a una descripción a partir de la lógica interna de los espacios construidos, un estudio desde dentro, y con ello establecer la forma básica o patrón formal invariante que se manifiesta en dicha construcción y a partir de él definir un Modelo Concreto Hipotético $(\mathrm{MCH})$ de la organización espacial de esa construcción. Este sería el primer nivel de análisis de un fenómeno, en el que se obtiene un modelo del que hay que comprobar su validez, lo cual es posible hacer mediante su comparación con otros modelos, tanto sincrónicos como diacrónicos (CRIADO, 1999, 2002).

Para llevar a cabo este análisis se debe aplicar un mecanismo de zoom. Es un modelo metodológico e interpretativo que se basa en la multidimensionalidad del espacio, las distintas escalas en las que se manifiesta una formación socio-cultural, permitiendo observar y comprender los rasgos formales en cada uno de los niveles identificados como «di- ferentes objetivaciones de los mismos principios o códigos estructurales que dan lugar a su/una regularidad espacial manifestada en la existencia de relaciones de compatibilidad entre los diferentes niveles y códigos espaciales» (CRIADO, 1999: 10).

Pero toda esta práctica no debe caer en saco roto, sino que debe servir como herramienta para una mejor gestión del patrimonio construido, una respuesta ante las demandas planteadas por nuestra sociedad ante la existencia del registro arquitectónico, una vía por la cual, partiendo de la investigación básica, sea posible facilitar la comprensión e interpretación del pasado, desacralizando la ruina arqueológica, el edificio histórico. La investigación básica debe ser el punto de partida de un proceso que culmina en la puesta en valor y divulgación del Patrimonio construido, pero ésta no se debe reducir a una mera práctica interpretativa, sino que ha de actuar como una técnica que por un lado, se adapta y responde a los problemas planteados por la existencia del Patrimonio (p.e. definir objetivos de restauración), y por otro, genera conocimiento que revierte en la propia sociedad (definir discurso explicativo del contexto social original de la construcción). A este respecto, se comparte una estrategia de investigación concreta que defiende la superación de esta falsa dicotomía entre investigación y gestión mediante la articulación de proyectos planteados como programas de Gestión Integral del Patrimonio, en los que el conocimiento generado por la disciplina arqueológica revierta en la sociedad mediante su revalorización y divulgación, en transformar el patrimonio en un recurso cultural (Criado, 1996a, 1996b; Criado, GonZÁlez, 1994, GONZÁLEZ, 1996).

\section{DE LA EXTRACCIÓN DEL MODELO AL SENTIDO MEGALÍTICO}

El programa de investigación propuesto se basa en la comparación de distintos modelos que, o bien se extraen del estudio de varios casos y varias dimensiones de un misma formación sociocultural, o de la comparación de modelos de distintas sociedades. Es un programa amplio y ambicioso, pues implica el estudio en singular de distintos ámbitos de un mismo fenómeno, y para ampliar el nivel de contrastación, incluso de otros fenómenos distintos. Por lo tanto, lo que aquí se presenta es un valoración de distintos modelos extraídos de estudios parciales, que no independientes, y que cobran auténtica significación cuando se llega a este nivel de análisis. Se trata de presentar cómo un mismo concepto de espacialidad subyace a la organización espacial de distintas dimensiones del megalitismo, cómo es posible compararlos y advertir que bajo sus múltiples manifestaciones todo parece responder a una misma idea. 

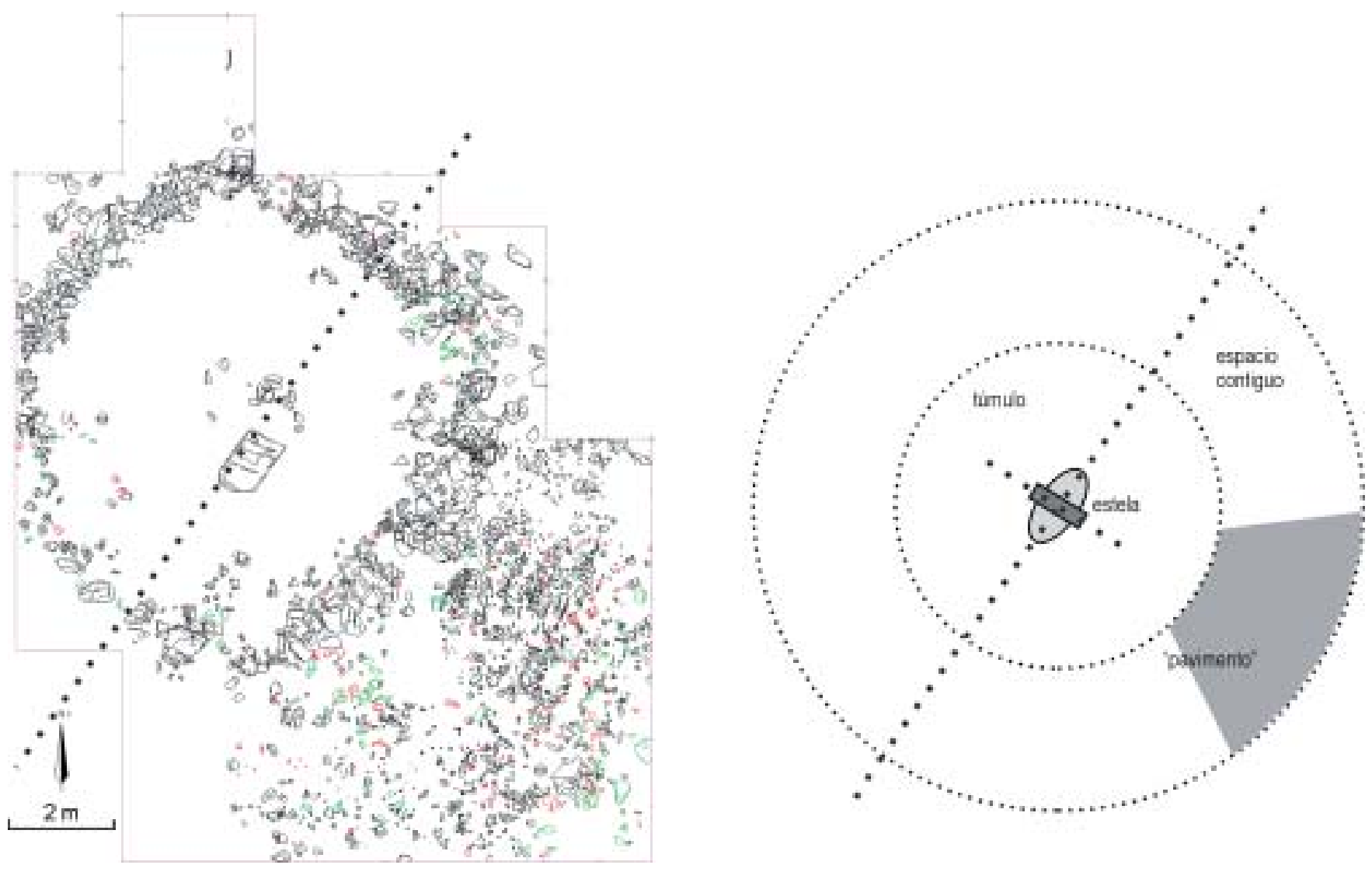

Fig. 1. Planta del túmulo n. 3 de San Cosme y esquema del modelo de sus distintos espacios

A partir de los distintos niveles de articulación del fenómeno megalítico, de sus distintas dimensiones espaciales, se comparan los modelos identificados en el ámbito arquitectónico funerario y en el ámbito del emplazamiento de estas primeras construcciones monumentales de la fachada atlántica europea. Con todo, un mayor nivel de comprobación de las hipótesis aquí presentadas se obtendría con la comparación de los modelos obtenidos del resto de evidencias formales del período, como la relacionada con los espacios de habitación, con la distribución de las representaciones gráficas en el interior de la cámara o en la superficie de un cacharro, etc.

Tras el análisis formal y perceptivo de un determinado aspecto del fenómeno (en este caso un túmulo), la primera fase del método pasa por identificar la forma básica de la construcción, el modelo al que responde (el Modelo Concreto Hipotético-MCH), en definitiva, la idea de la que ha partido. En el caso de la mámoa n. ${ }^{\circ} 3$ del Alto de San Cosme, el modelo espacial definido por el túmulo $(\mathrm{MCH})$ es el de un espacio de forma circular, cerrado, con un punto central dominante en torno al cual se organiza, y por el cual pasa un eje que divide al túmulo en dos mitades de ca- racterísticas diferentes (Figura 1), ya que mientras la mitad sudeste tiene un anillo más amplio y una litología más variada (en la que destacan las piedras verdes y blancas, visualmente más llamativas), la noroeste es mucho más somera, con el anillo menos potente (incluso una única hilada de piedras), con una menor cantidad de estas piedras destacadas $^{3}$.

En el propio yacimiento tenemos otros ámbitos en los que podemos identificar el modelo espacial implementado, y así empezar la fase de validación del modelo propuesto para el túmulo, para con ello llegar al Modelo Concreto Ideal (MCI) del mismo. Uno de ellos es el espacio circundante, del que, aunque se excavó una pequeña porción se puede advertir que solamente se ha realizado cierto esfuerzo constructivo en la mitad sudeste del entorno del túmulo, ya que en la zona opuesta no se documentó ninguna evidencia de este estilo. Esto muestra dos mitades disimétricas que están organizadas en torno al eje marcado por el túmulo (Figura 1).

\footnotetext{
${ }^{3}$ El análisis de este túmulo y sus distintos espacios se encuentra publicado en Mañana et alii. 2002.
} 

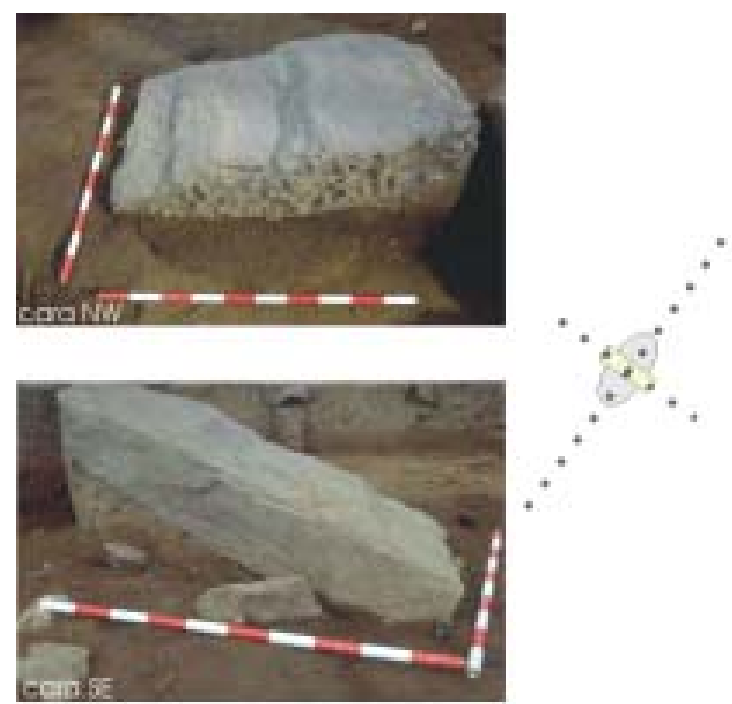

Plants de Cas:

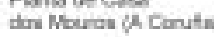

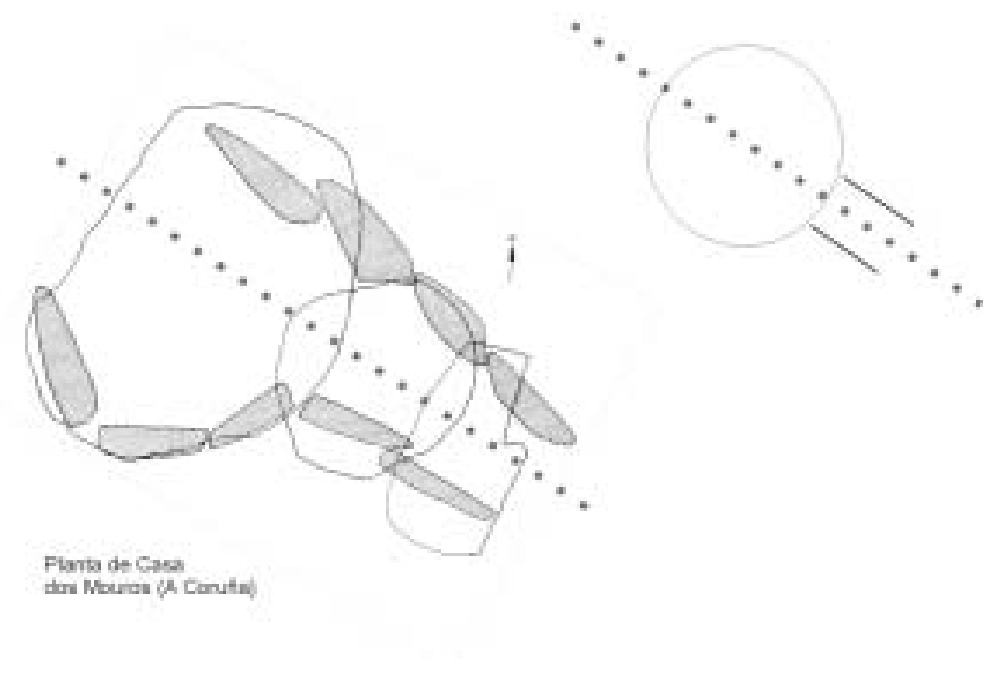

Fig. 2. Esquema del modelo espacial del espacio central: la estela y la cámara

El otro espacio que se configura en este yacimiento es el central, donde se encuentra la estela, que en sí misma es formalmente disimétrica, ya que mientras una de las caras menores es lisa (la sudeste), la opuesta es rugosa, confirmando el eje de organización del túmulo y del espacio circundante (Figura 2). Se situaría en posición central dentro de lo que sería el espacio de enterramiento, perpendicular al eje de organización identificado a nivel del túmulo y con cada uno de sus lados disimétricos hacia esas dos mitades.

El método interpretativo propuesto para identificar y validar las regularidades espaciales dentro del fenómeno megalítico debe buscar más ejemplos de configuración espacial, y ya no sólo en lo relativo a este yacimiento o a este tipo de manifestación del megalitismo, sino en relación con todo el fenómeno cultural. Así, cogiendo como ejemplo la configuración del espacio interior de uno de los grandes dólmenes de la provincia de A Coruña, la cámara de Casa dos Mouros, podemos advertir que, a pesar de faltarle al menos una losa de la parte de cabecera, es formalmente disimétrica: la cámara tiene las losas de uno de sus lados dispuestas linealmente, mientras que las del lado opuesto están formando un arco; además, el centro de la cámara no está orientado respecto al eje que marca el corredor, lo que parece marcar cierta intención de no-simetría (figura 2).

Así, siguiendo la disciplina del método interpretativo referida en este texto (CRIADO, 1999), se ha analizado en primer lugar un ámbito específico del tema propuesto en sus diferentes escalas, que en este caso ha sido la arquitectura del túmulo. Conviene no sólo analizar el resultado final, sino el proceso constructivo o cadena tecnológicooperativa $^{4}$ que da lugar a él. Esta aproximación permitirá identificar las diferentes opciones constructivas y compro- bar hasta qué punto están determinadas por el modelo espacial subyacente, por imperativo constructivo o por razones funcionales ${ }^{5}$. Así, en el caso del túmulo $n .^{\circ} 5$ de Forno dos Mouros (Ortigueira, A Coruña) ${ }^{6}$, en el que se documentó una cámara en muy buen estado de conservación, se advierte que la disposición de las losas según el tipo de material (cuarzo, pizarra, cuarcita) es formalmente opuesto, combinando losas de características (color-textura) distintas e incluso opuestas (pizarra: oscuro y liso; cuarzo: claro y rugoso): el corredor es exclusivamente de cuarzo; en la cámara, el lado $\mathrm{W}$ es de pizarra-cuarcita mientras que en el E predomina el cuarzo-cuarcita; la losa de cabecera, en el fondo de la cámara, de pizarra, se opone a los cuarzos del corredor (Figura 3). De aquí se deriva el Modelo Concreto Ideal (MCI), que en realidad es la síntesis de los $\mathrm{MCH}$ de cada nivel analizado y que define la regularidad espacial que subyace a los diferentes niveles de la arquitectura megalítica.

En tercer lugar se compara el modelo anterior con el patrón del emplazamiento tumular, aspecto que a priori es el que mejor ilustra las formas de organización del paisaje megalítico. Se consideran así las distintas escalas de articulación del emplazamiento tumular, desde el rango de detalle al comarcal, su correspondencia con el medio físico y la

${ }^{4}$ V. la definición de este concepto en Prieto (1999) y Cobas y Prieto (2002).

5 No es frecuente todavía aplicar la cadena operativa a la arquitectura. Quirós (1993) es una excepción.

6 Modelo basado en la excavación realizada en 2001 por la Fundación Federico Maciñeira (Ortigueira-A Coruña); las imágenes se corresponden a un modelo virtual demostrador realizado por Boado Integra. 


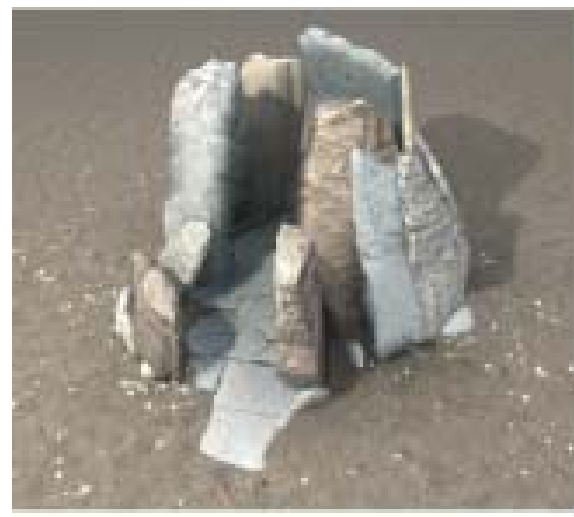

Construoción de la comara y eriosodo

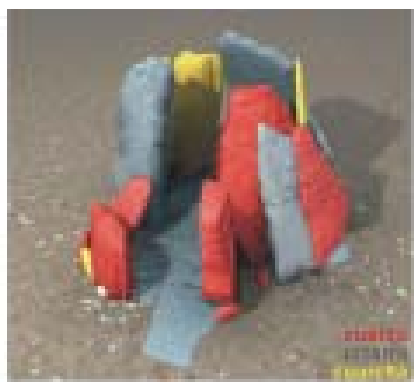

Representación de los datmioe materiaies empleados

Fig. 3. Ejemplo del proceso constructivo de un túmulo, basado en los datos de la excavación del túmulo n. 5 de Forno dos Mouros (Ortigueira, A Coruña)

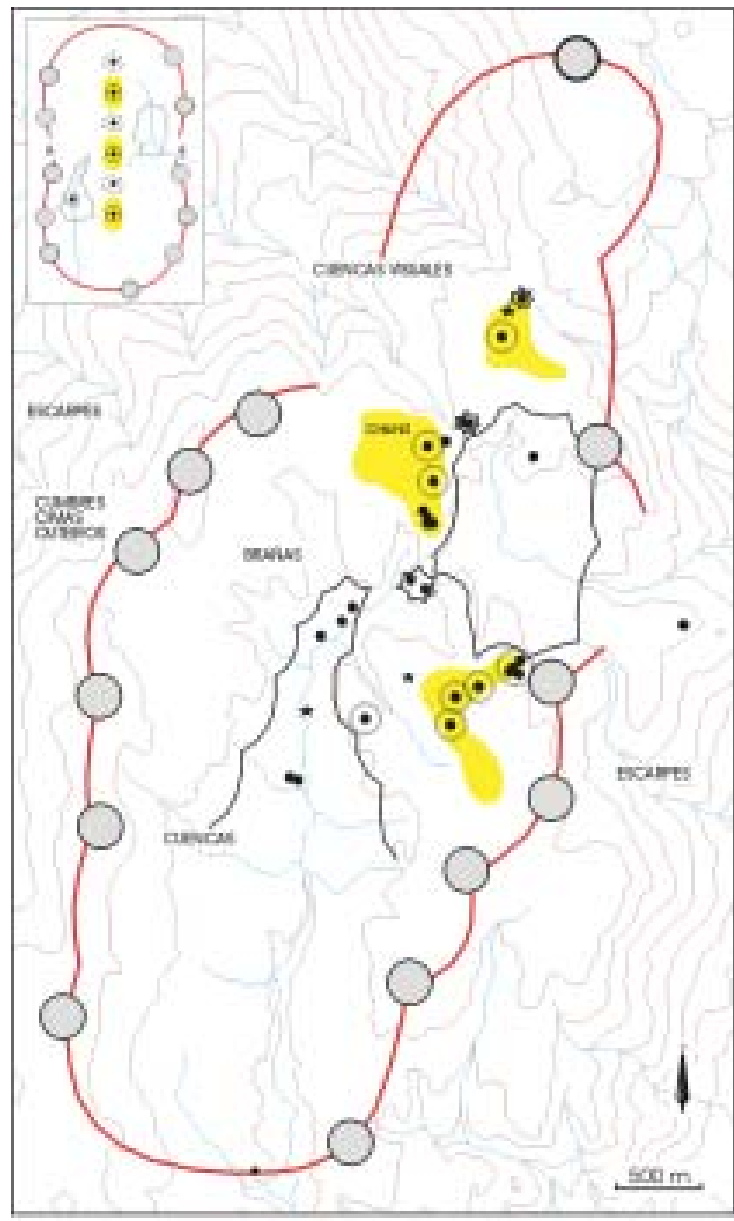

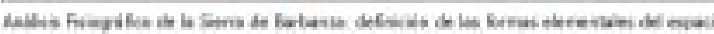

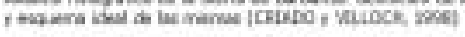

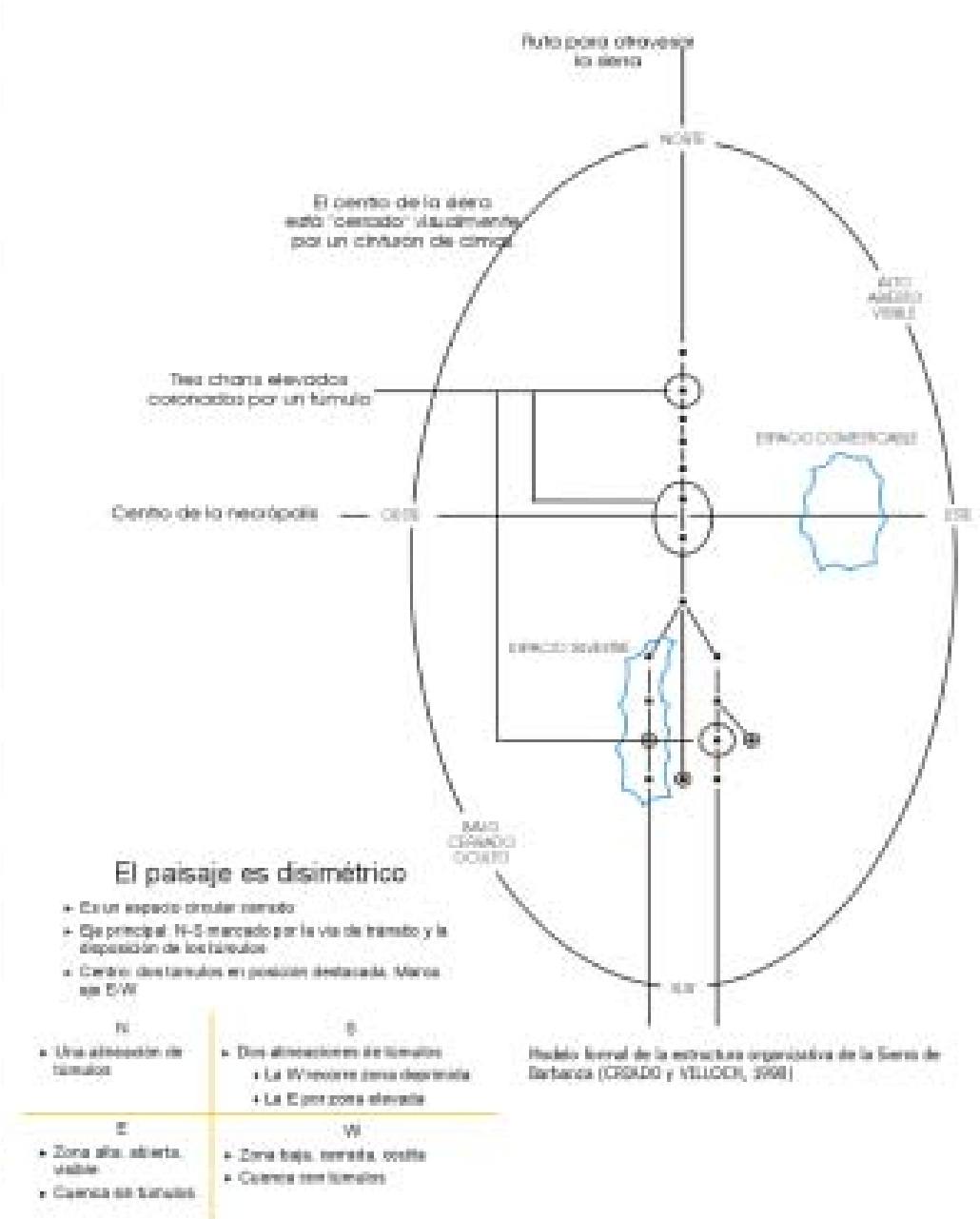

Fig. 4. El modelo espacial del paisaje en la Sierra de Barbanza 
fisiografía, etc (Criado, Villoch, 1998). De aquí se desprende el Modelo Genérico Hipotético del espacio megalítico (Figura 4$)^{7}$.

En cuarto lugar se compara ese modelo con los esquemas formales derivados de otros ámbitos culturales o fenoménicos; pueden ser: el espacio doméstico, el uso del suelo, la territorialidad y sus formas, o incluso otras zonas distintas. Las correspondencias entre los diferentes códigos permiten transformar lo "hipotético» en «ideal», definir así el Modelo Genérico Ideal y describir lo que de hecho constituye el código estructural del paisaje monumental, entendido como el modelo ideal que da cuenta del paisaje cultural del contexto considerado y establece las correlaciones formales entre los diferentes niveles o aspectos que comprenden ese fenómeno.

El análisis muestra la recurrencia de un mismo código estructural del espacio en diferentes niveles y ámbitos de la realidad. Su reaparición en dominios empíricos diferentes, aunque pertenecientes a un mismo contexto cultural, nos muestra la pertinencia de la hipótesis interpretativa. El hecho en sí de haber aislado esta hipótesis (ie, de forma estricta: haber descubierto que hay buenas razones para creer que la arquitectura megalítica en sus diferentes niveles está organizada en función de una misma estructura espacial) constituye ya una interpretación blanda.

El modelo espacial identificado para este fenómeno se puede definir por la creación de un espacio circular, cerrado, con un punto central dominante, que marca un eje en torno al cual se organiza el espacio en dos mitades disimétricas, estando la mitad sudeste más trabajada, más «humanizada» mientras que la noroeste es mucho más somera, tiene un menor esfuerzo constructivo, identificándose en todo caso una apropiación y significación de elementos naturales, lo que a priori implica una gran comprensión del entorno.

Nos encontramos ante un proceso que se basa en la modificación del entorno, en la que se hace visible y permanente la muerte al construirse monumentalmente un referente de control y manipulación del espacio y del tiempo. El círculo, la percepción circular que se observa en todos los niveles de análisis, tiene implicaciones de control del entorno, lo que es relacionable con cuestiones propias de la domesticación del entorno. En los procesos constructivos,

\footnotetext{
7 No podemos negar que esta aproximación adolece, a los ojos de la persona que desconoce el registro que utilizamos, de una aparente orientación empírico-fenomenológica, pues parecería que vamos saltando de ejemplo en ejemplo buscando los más oportunos para nuestro estudio. En realidad estos ejemplos representan tendencias generales, en las que, por la brevedad de este texto, no podemos entrar.
}

en sus características perceptivas y formales, se identifica también el carácter ambiguo de este fenómeno: es disimétrico formal y visualmente, ya que en todos los ámbitos analizados se contraponen una zona más natural, menos humanizado (la noroeste) con otra en la que se concentra la actividad humana, que se configura como menos natural (sudeste). A nivel constructivo también se reproduce esta intención de ambigüedad, ya que a la vez que se construye, se destruye o se oculta, pero con un proceso que implica la monumentalización de la construcción (la construcción del túmulo supone la ocultación de la cámara, y en muchos casos el cierre para el uso de este espacio de enterramiento).

Para ir más allá en el proceso interpretativo (esto es, para interpretar la hipótesis anterior y descubrir su sentido cultural), disponemos de dos opciones (o más, si fuera posible), que pueden (y deben) ser utilizadas simultáneamente (CRIADO, 1999):

— «leer» la hipótesis estructural desde un patrón de racionalidad que ofrezca un marco contextual de comprensión,

— «contrastar» el modelo estructural que hemos desprendido del análisis con el obtenido al estudiar un fenómeno distinto pero relacionado.

Por ejemplo contrastar el modelo del paisaje megalítico de una zona de Galicia con el del paisaje doméstico que le sucede en la misma zona en la Edad del Bronce (CRIADO, 1999): el espacio doméstico, uso del suelo y territorialidad de la Edad del Bronce, la distribución y organización de los petroglifos (arte rupestre al aire libre)... De este modo, valorando la dinámica de continuidad y discontinuidad en las formas de paisaje, nos preguntamos qué significan las permanencias y los cambios y podemos interpretar el modus operandi y el sentido de las estrategias megalíticas de domesticación del espacio físico y construcción de un paisaje cultural. Debemos resaltar que el sentido se desprende tanto del hecho de que ese código estructural se mantenga en épocas distintas, como del hecho de resultar contradictorio con el registro de esos otros momentos y apreciamos una historia de continuidades y rupturas, pues se mantiene una misma «arquitectura» general del paisaje y al mismo tiempo se modifica el modelo anterior. La diferencia más obvia entre ambos momentos es la domesticación efectiva de un paisaje que, en el modelo megalítico, era de carácter fundamentalmente ritual. En cualquier caso, hemos pasado a una interpretación fuerte.

Ahora bien, hay una diferencia entre definir una regularidad formal en la arquitectura megalítica, comprobar que esa regularidad forma parte del código estructural del megalitismo como paisaje cultural, e interpretar el sentido de ese 
código (CRIADO, 1999, 2002). Lo primero y lo segundo se consigue mediante una estrategia fundamentalmente formalista (un análisis formal y un método formalizado) sin poner en juego valoraciones subjetivas. Pero todavía no se ha descubierto el sentido Arqueo-lógico; para ello se debe introducir un principio de inteligibilidad exterior que en nuestro caso optamos por bajarlo desde un modelo de racionalidad antropológico.

Tal y como ya se ha tratado en otros puntos (CRIADO, $1988,1989,1993)$, en el megalitismo se inicia un proceso creciente de domesticación del entorno que no sólo es expresión de una nueva economía y aparato tecnológico, sino ante todo de una nueva relación de la sociedad con la naturaleza, caracterizada por una actitud activa ante ella que se aplica a su transformación sistemática y progresiva. Esta actitud expresa una fisura radical en el orden de la cultura y se corresponde con la sustitución de un patrón de racionalidad anterior, identificado con el pensamiento salvaje de Lévi-Strauss, por otro que ha sido denominado racionalidad doméstica, que inaugura el proceso de modificación y explotación del espacio físico y se correlaciona con transformaciones paralelas en la sociedad que se corresponden con la modificación y (muy pronto) explotación también del cuerpo social.

Esta nueva forma de estar-en-el-mundo implica no sólo una nueva forma de relacionarse con la naturaleza, sino también de conceptualizar el espacio y el tiempo. El paisaje social de este momento sería la expresión y materialización práctica de ese universo conceptual. En concreto, la aparición de la arquitectura monumental sería la representación de esa nueva forma de estar-en-el-mundo. La arquitectura es una tecnología de construcción del paisaje social que opera la domesticación del mundo físico a través de dispositivos artificiales no sólo introduciendo hitos arquitectónicos en el espacio natural para ordenarlo según referencias culturales, sino también controlando e imponiendo un determinado patrón de percepción del entorno a los individuos, una pauta para experimentar el espacio-tiempo comunitario e individual.

La arquitectura megalítica (a través de todos sus niveles fenoménicos, desde la cámara y el túmulo a la organización de la necrópolis y la distribución de los monumentos en el territorio) construye un modelo de pensar el mundo que es también una forma de habitarlo, de estar en él. Es un modelo basado en una artificialización que, por ser incipiente, se cierra en círculos de actividad humana rodeados por un entorno silvestre. El círculo tiene un centro substantivado por lo funerario, la muerte, el más allá; dos mitades opuestas, una hacia naciente (sobre todo al SE) vinculada a la vida y al mundo humano (seguramente también al doméstico $)^{8}$, y otra hacia poniente relacionada con la naturaleza, lo silvestre, lo salvaje. La luz desempeña un papel esencial en esta oposición, siendo la mitad de oriente luminosa y blanca, y la mitad opuesta oscura y negra ${ }^{9}$. No es imposible pensar que el itinerario norte-sur (el que se corresponde con el eje que divide las dos mitades) fuese en la tierra una ruta procesional ${ }^{10}$ que reproducía un itinerario en el firmamento de un valor cosmológico que se nos escapa.

Ciertamente se ha pasado de la interpretación débil (constatar la existencia de una regularidad formal) a la interpretación fuerte (reconstruir narrativamente un sentido).

Para interpretar la materialización de un patrón de racionalidad a través de la monumentalidad no sólo debemos fijarnos en quién puede asumir los costes de construir un monumento o a quién beneficia que el grupo lo asuma. Esto permitiría explicar la función social de un monumento concreto, pero para interpretar su sentido cultural es preciso reconocer que a través de esa estrategia de materialización se expresa una racionalidad cultural concreta que implica, entre otras cosas, una forma específica de construir la realidad social basándose en nuevos conceptos de espacio, tiempo y de interrelación sociedad-naturaleza.

\section{Bibliografía}

Criado Boado F., 1988, Arqueología del Paisaje y Espacio Megalítico, Arqueología Espacial, n. ${ }^{\circ} 12$, pp. 61-117.

Criado Boado F., 1989, Megalitos, Espacio, Pensamiento, Trabajos de Prehistoria n. ${ }^{\circ}$ 46, pp. 75-98.

CRIADO BOAdo F., 1993, Visibilidad e interpretación del registro arqueológico, Trabajos de Prehistoria, n. ${ }^{\circ}$ 50, pp. 39-56.

CRIADO BOADO F., 1996a, La arqueología del paisaje como programa de gestión integral del patrimonio arqueológico, Boletín del Instituto Andaluz del Patrimonio Histórico, n. ${ }^{\circ}$ 14, pp. 15-19.

Criado BOADO F., 1996b, Hacia un modelo integrado de investigación y gestión del Patrimonio Histórico: la cadena interpretativa como propuesta, Boletín del Instituto Andaluz del Patrimonio Histórico, n. ${ }^{\circ} 16$, pp. 73-9.

Criado BoAdo F., 1999, Del Terreno al Espacio: Planteamientos y Perspectivas para la Arqueología del Paisaje, CAPA (Criterios y Convenciones en Arqueología del Paisaje), n. ${ }^{\circ}$ 6, Santiago.

8 Es el lado hacia el que quedan los asentamientos domésticos visibles desde el monumento, los espacios factibles de uso humano y, según se ha comprobado en ciertos casos, efectivamente aprovechados.

9 Denotado por la orientación de los corredores hacia el SE, las diferentes condiciones de iluminación de la mitades norte y sur de las cámaras en función de la ligera disimetría que éstas tienen y, sobre todo, por el empleo de materiales geológicos distintos en las cámaras y corazas tumulares, con predominio del cuarzo hacia el este y de la pizarra o piedras oscuras hacia el oeste.

${ }_{10}$ Pues es la orientación esencial que presentan las necrópolis, estando subrayada por el hecho de que el tránsito y relaciones visuales entre túmulos se ajusta a esa ruta. 
Criado Boado F., 2002, Arqueológicas. La Razón Perdida, Madrid.

Criado BoAdo F., GonZÁlez MéNDEZ M. 1994, La puesta en valor del patrimonio arqueológico desde la perspectiva de la arqueología del paisaje, en VV.AA., Conservación arqueológica. Reflexión y debate sobre teoría y práctica, Cuadernos del Instituto Andaluz del Patrimonio Histórico, III, pp. 58-75.

Criado Boado F., Villoch VÁzquez V., 1998, La monumentalización del paisaje: percepción y sentido original en el megalitismo de la Sierra de Barbanza (Galicia), Trabajos de Prehistoria, n. ${ }^{\circ} 55$ (1), pp. 63-80.

Cobas Fernández M.I., Prieto Martínez M.P., 2002, The technological chain as a methodological and theoretical tool from archaeology, XIVth Congress of the UISPP (Liége, Belguique, september 2001), Oxford, en prensa.

GONZÁLEZ MÉNDEZ M., 1996, El ocio y el reciclado: la conversión del vestigio arqueológico en producto de consumo, Boletín del Instituto Andaluz del Patrimonio Histórico, n. ${ }^{0}$ 14, pp. 24-7.

Hillier B., Hanson J., 1984, The social logic of space, Cambridge.

Jones C., Hayden C. (eds.), 1998, The Archaeology of Perception and the Senses, Archaeological Review from Cambridge, n. ${ }^{\circ} 15$ (1), Cambridge.

LOCOCK M., 1994, Meaningful Architecture, en Locock M. (ed.), Meaningful Architecture: social interpretations of buildings, pp. 1-13. Avebury.

Mañana BorrazÁs P., Blanco Rotea R., Ayán Vila J.M., 2002, Arqueotectura 1: Bases teórico-Metodológicas para una Arqueología de la Arquitectura, TAPA (Traballos en Arqueoloxía da Paisaxe), n. ${ }^{\circ} 15$. Santiago de Compostela: Laboratorio de Arqueoloxía (CSIC-XuGA).

Prieto Martínez M.P, 1999, Caracterización del estilo cerámico de la Edad de Bronce en Galicia: carámica campaniforme y cerámica no decorada, Complutum, n. ${ }^{\circ}$ 10, pp. 71-90.

Quirós CAstillo J.A., 1993, Produzioni di laterizi nell'Alta Valdinievole: la Valleriana (XVII-XX sec.), en Atti del Convegno su Pluriattività e Mercati in Valdinievole (XVI-XIX secolo), pp.139-47. Buggiano.

Shanks M., Tilley C., 1987, Social Theory and Archaeology,. Cambridge. 